\title{
Analytical Modeling of Distributed Location Based Access for Vehicular Ad Hoc Networks
}

\author{
F. J. Martin-Vega*, B. Soret ${ }^{\dagger}$, M. C. Aguayo-Torres*, G. Gomez* and I. Z. Kovacs ${ }^{\dagger}$ \\ ${ }^{*}$ Dpto. Ingeniería de Comunicaciones, Universidad de Málaga (Spain) \\ Email: fjmvega@ic.uma.es \\ ${ }^{\dagger}$ Nokia Bell Labs
}

\begin{abstract}
One of the key ingredients of Intelligent Transportation Systems (ITS) is delivery of broadcast status messages among vehicles for safety purposes. This requires an efficient Medium Access Control (MAC) that provides low average delay and high reliability. To this end, Carrier Sense Multiple Access (CSMA) has been commonly proposed for Vehicle Ad Hoc Networks (VANETs). Nevertheless, the hidden-node problem can jeopardize the reliability of CSMA, whereas the latency when accessing the channel can be unbounded. To overcome these limitations, resource allocation based on the geo-location of the vehicles can be applied in VANETs. For example, a distributed location based access (DLOC) algorithm has been proposed such that vehicles access orthogonal resource blocks based on their position, aiming at maximizing the distance of co-channel transmitters. In this paper we propose a stochastic geometry approach to analyze DLOC taking into account path loss and fading as well as the random location of transmitting vehicles. Analytical results include the average interference, average binary rate and capture probability, i.e. probability of successful message transmission. It is shown that increasing the number of RBs increases reliability but there is a trade off between reliability and average BR.
\end{abstract}

\section{INTRODUCTION}

Vehicle awareness is one of the enablers of Intelligent Transportation Systems (ITS) to increase safety on the roads. In such approach each vehicle periodically transmits broadcast status messages informing nearby vehicles of its position, velocity and direction, among other parameters. These broadcast messages require high reliability and low latency thus making the design of the Medium Access Control (MAC) layer an issue of paramount importance. Distributed solutions, like slotted ALOHA, have been evolved into listen-before-talk solutions aiming to avoid collisions of nearby transmitting nodes. However, this sensing does not completely remove collisions due to the hidden node problem.

A novel MAC technique is Distributed Location (DLOC) based access [1], where vehicles access the channel based on its position. The road is divided into segments, where each segment is associated with an orthogonal Resource Block (RB). Hence each vehicle transmits its related broadcast status message using a single $\mathrm{RB}$, which is associated with its current position. The mapping between RBs and segments is made to maximize the co-channel distance. The benefits of this technique are: (i) high reliability, since the distance to interfering vehicles can be increased with the number of RBs and (ii) MAC layer does not add any delay on accessing the channel, i.e. vehicles start their transmission once they have data to transmit.

Building on [1], analytical models can provide more insight of the inter-plays among reliability, binary rate and number of RBs for medium access. In this context, Stochastic Geometry [2] is a promising tool for performance analysis that allows tractable and realistic analysis due to the random nature of transmitting vehicules in VANETs. [3] analyzes the capture probability, average throughput using ideal adaptive modulation and coding and mean density progress of transmitted packets for the case of unicast transmissions with ALOHA. The case of a head vehicle that broadcasts info and control messages to a sectorized cluster of client vehicles is considered in [4]. This work models the positions of vehicles as a Cox process whose density follows a Fox distribution; however, the interference caused by other transmitting vehicles is not taken into account. In this work DLOC access technique is analyzed using the single lane abstraction model where positions of transmitting vehicles are modeled as points of a Poisson Point Process (PPP) in the real line. Considered performance metrics include the complementary cumulative density function (ccdf) of the Signal to Interference plus Noise Ratio (SINR), i.e. the capture probability, the average interference, and the average Binary Rate (BR) considering fixed modulation.

The rest of the paper is organized as follows. Section II presents the system model. The mathematical analysis is depicted in Section III. Finally, numerical results are illustrated in Section IV, whereas conclusions are drawn in Section V.

\section{SYSTEM MODEL}

It is considered a straight road with several lanes as appears in Fig. 1, where the $z$ coordinate is neglected. Hence positions of vehicles are assumed to follow a uniform PPP $\Phi=$ $\left\{\mathrm{V}_{0}, \mathrm{~V}_{1}, \cdots\right\} \subset \mathbb{R}$ with density $\lambda$. It is assumed that at a given time instant a vehicle has data to transmit with probability $p_{\mathrm{a}}$, hence the set of active vehicles $\Phi^{(\mathrm{a})}=\left\{\mathrm{VT}_{0}, \mathrm{VT}_{1}, \cdots\right\}$ is obtained through independent thinning from $\Phi$ and its density is $\lambda p_{\mathrm{a}}$. The road is divided into segments of length $d_{\mathcal{A}}$ meters, and each segment is associated with a given orthogonal RB, being $n_{\mathrm{RB}}$ the number of RBs in the system. The mapping between segments and RBs is made with maximum co-channel distance criterion; a sample of the mapping is shown in Fig. 1 for the case of three RBs. The set of contiguous segments using different RBs is called cluster in this paper. Symbol 


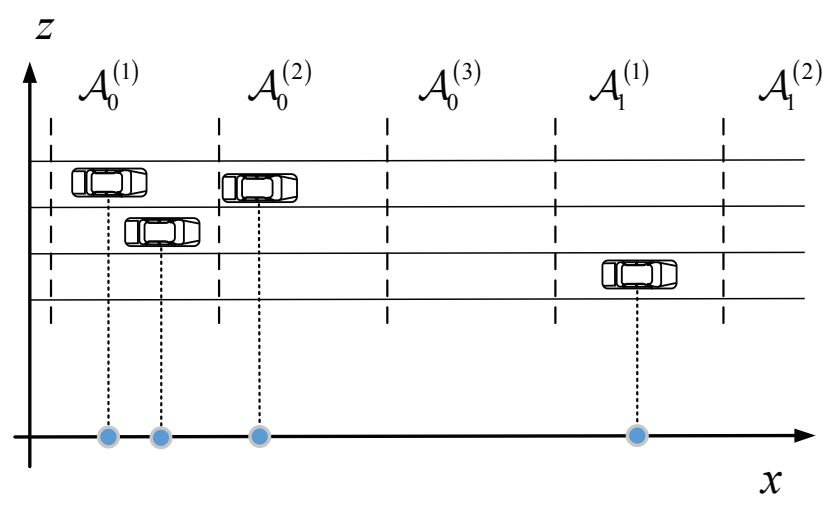

Fig. 1. Sketch of distributed location based access with 3 RBs for a multilane road using segments that include portions of several lanes. On the bottom it is shown a single lane abstraction model where positions of vehicles are treated as points in the real line.

$\mathcal{A}_{c}^{\left(\mathrm{RB}_{j}\right)}$ identifies the segment associated with $\mathrm{RB}_{j}$ within cluster $c$. The set that represents all the segments associated with $\mathrm{RB}_{j}$ is represented as $\mathcal{A}^{\left(\mathrm{RB}_{j}\right)}=\bigcup_{c=-\infty}^{\infty} \mathcal{A}_{c}^{\left(\mathrm{RB}_{j}\right)}$.

The analysis is performed for the typical transmitter, i.e. a randomly selected Vehicle Transmitter (VT). This transmitter is named the probe VT, and it is represented with symbol $\mathrm{VT}_{0}$. In this paper we made an abuse of notation since $\mathrm{VT}_{0}$ is used to represent the probe VT as well as its position in the real line. Analogously its associated RB is the probe $\mathrm{RB}$, which is denoted by $\mathrm{RB}_{0}$. Fig. 2 shows a sketch of the system model. Since PPP are translation invariant Point Processes (PP), it is considered that the probe segment, $\mathcal{A}_{0}^{(\mathrm{RB})}$, is centered at the origin. Hence the position of the probe $\mathrm{VT}, \mathrm{VT}_{0}$, is uniformly distributed within the interval $\left[-d_{\mathcal{A}} / 2, d_{\mathcal{A}} / 2\right)$. Being the probe segment centered at the origin, the $c$-th co-channel segment, $\mathcal{A}_{c}^{\left(\mathrm{RB}_{0}\right)}$, can be expressed as $\mathcal{A}_{c}^{\left(\mathrm{RB}_{0}\right)}=\left\{y \in \mathbb{R}: c n_{\mathrm{RB}} d_{\mathcal{A}}-\frac{d_{\mathcal{A}}}{2} \leq y<c n_{\mathrm{RB}} d_{\mathcal{A}}+\frac{d_{\mathcal{A}}}{2}\right\}$. The set of co-channel segments are centered around $c \cdot n_{\mathrm{RB}} \cdot d_{\mathcal{A}}$ with $c \in \mathbb{Z}$. Transmitted signals suffer from Rayleigh fading, hence the channel power gain is exponentially distributed with unitary mean. Path loss is modeled through a path loss slope $\tau$ and a path loss exponent $\alpha$. Having a receiver placed at location $x$, the SINR can be expressed as follows

$$
\operatorname{SINR}(x)=\frac{H_{\mathrm{VT}_{0}}\left(\tau\left|\mathrm{VT}_{0}-x\right|\right)^{-\alpha} \rho_{\mathrm{VT}}}{I(x)+\sigma_{n}^{2}}
$$

where $|\cdot|$ the Euclidean distance, $H_{\mathrm{VT}_{0}}$ is the fading power gain between $\mathrm{VT}_{0}$ and the test receiver, $\rho_{\mathrm{VT}}$ is the transmit power per $\mathrm{Hz}, \sigma_{n}^{2}$ is the noise power and $I(x)$ the received interference at $x$. It is assumed that there is a maximum communication range given by $d_{\max }$, hence a receiver cannot detect signals from transmitters farther than $d_{\max }$.

Thus the interference term can be expressed as follows

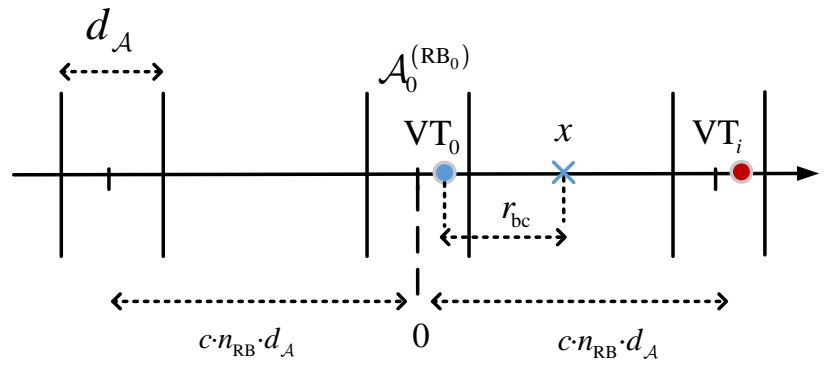

Fig. 2. Illustration of co-channel segments. The probe transmitter is represented as $\mathrm{VT}_{0}$, the probe $\mathrm{RB}$ and segment as $\mathrm{RB}_{0}$ and $\mathcal{A}_{0}^{\mathrm{RB}}{ }_{0}$ respectively, the probe receiver as a blue $x$ and a single interfering vehicle as $\mathrm{VT}_{i}$. The length of each segment is represented as $d_{\mathcal{A}}$ and thus $n_{\mathrm{RB}} \cdot d_{\mathcal{A}}$ is the minimum co-channel distance.

$$
\begin{aligned}
& I(x)=\sum_{\mathrm{VT}_{i} \in \Phi^{(\mathrm{a})} \backslash\left\{\mathrm{VT}_{0}\right\}} H_{\mathrm{VT}_{i}}\left(\tau\left|\mathrm{VT}_{i}-x\right|\right)^{-\alpha} \\
& \times \rho_{\mathrm{VT}} \mathbf{1}\left(\mathrm{VT}_{i} \in \mathcal{A}^{\left(\mathrm{RB}_{0}\right)}\right) \mathbf{1}\left(\mathrm{VT}_{i} \in \mathfrak{b}_{x}\left(d_{\text {max }}\right)\right)
\end{aligned}
$$

being $\mathbf{1}(\cdot)$ the indicator function and $\mathfrak{b}_{x}\left(d_{\max }\right)$ the ball centered at $x$ with radius $d_{\text {max }} . H_{\mathrm{VT}_{i}}$ is the fading between $\mathrm{VT}_{i}$ and $x$. The capture probability represents the probability that a message is correctly received. Having a receiver placed at location $x$, it is expressed as the probability of the SINR being higher than a given threshold, which is the ccdf of the SINR. The useful bandwidth, $b_{w}$, is equally divided among all RBs and transmissions use fixed modulation and coding. Hence, using the Shannon's formula, the BR of correctly received bits can be expressed as [5]

$$
\operatorname{BR}(x)=\mathbf{1}(\operatorname{SINR}(x)>\gamma) \cdot \frac{b_{w}}{n_{\mathrm{RB}}} \cdot \log _{2}(1+\gamma)
$$

where $\gamma$ is the SINR threshold associated with the used modulation and coding scheme.

\section{Performance Analysis}

The studied scenario relies on transmission of broadcast status messages, and therefore the receiver is placed at a distance $r_{\mathrm{bc}}$ from the probe transmitter $\mathrm{VT}_{0}$. In other words, the metrics of interest - capture probability and average BR are evaluated at $x=\mathrm{VT}_{0}+r_{\mathrm{bc}}$.

In order to obtain the capture probability, it is necessary to compute the Laplace transform of the interference, which is given with the following lemma

Lemma 1. The Laplace transform of the interference evaluated at $x \in \mathbb{R}$ can be written as appears below

$$
\mathcal{L}_{I(x)}(s)=\exp \left(-\lambda \cdot p_{a} \sum_{c=-\left\lfloor d_{\max } /\left(n_{R B} d_{\mathcal{A}}\right)\right\rfloor}^{\left\lceil d_{\max } /\left(n_{R B} d_{\mathcal{A}}\right)\right\rceil} \kappa(c, s, x)\right)
$$

being the function $\kappa(c, s, x)^{1}$ expressed as appears in (4) where ${ }_{2} F_{1}(\cdot, \cdot, \cdot, \cdot)$ is the Gauss hypergeometric function and

\footnotetext{
${ }^{1}$ The dependence of functions $\mu_{L}^{(j)}(c, x)$ and $\mu_{U}^{(j)}(c, x)$ with $c$ and $x$ has not been written in (4), (6) and (7) for convenience.
} 


$$
\kappa(c, s, x)=\sum_{j \in\{1,2\}} \mathbf{1}\left(\mu_{L}^{(j)}<\mu_{U}^{(j)}\right)\left(\mu_{U}^{(j)}{ }_{2} F_{1}\left(1, \frac{1}{\alpha}, 1+\frac{1}{\alpha}, \frac{\left(\tau \mu_{U}^{(j)}\right)^{\alpha}}{-s \cdot \rho_{\mathrm{VT}}}\right)-\mu_{L}^{(j)}{ }_{2} F_{1}\left(1, \frac{1}{\alpha}, 1+\frac{1}{\alpha}, \frac{\left(\tau \mu_{L}^{(j)}\right)^{\alpha}}{-s \cdot \rho_{\mathrm{VT}}}\right)\right)
$$

$$
\begin{aligned}
& \mu_{U}^{(1)}=\min \left(c \cdot n_{\mathrm{RB}} \cdot d_{\mathcal{A}}+\frac{d_{\mathcal{A}}}{2}-x, d_{\max }\right) \\
& \mu_{L}^{(2)}=\max \left(c \cdot n_{\mathrm{RB}} \cdot d_{\mathcal{A}}-\frac{d_{\mathcal{A}}}{2}-x,-d_{\max }\right) \\
& \mu_{L}^{(1)}=\max \left(\mu_{L}^{(2)}, 0\right) ; \mu_{U}^{(2)}=\min \left(\mu_{U}^{(1)}, 0\right)
\end{aligned}
$$

Proof. The Laplace of the interference can be obtained as follows

$$
\begin{aligned}
& \mathcal{L}_{I(x)}(s)=\mathbb{E}_{I(x)}\left[\mathrm{e}^{-s I(x)]}\right. \\
& =\exp \left(-\lambda \cdot p_{a} \sum_{c=-\infty}^{\infty} \int_{y=c \cdot n_{\mathrm{RB}} \cdot d_{\mathcal{A}}-\frac{d_{\mathcal{A}}}{2}}^{c \cdot n_{\mathrm{RB}} \cdot d_{\mathcal{A}}+\frac{d_{\mathcal{A}}}{2}}\right. \\
& \left.\quad \frac{s(\tau|y-x|)^{-\alpha} \rho_{\mathrm{VT}}}{1+s(\tau|y-x|)^{-\alpha} \rho_{\mathrm{VT}}} \mathbf{1}\left(y \in \mathfrak{b}_{x}\left(d_{\max }\right)\right) \mathrm{d} y\right)
\end{aligned}
$$

where it has been expressed the exponential of the summation that defines the interference as a product over the PPP $\Phi^{(a)}$; it has been applied the Probability Generating Functional (PGFL) [2] of the PPP; it has been performed expectation over the fading and it has been expressed the region $\mathcal{A}^{\left(\mathrm{RB}_{0}\right)}$ as a summation of co-channel segments. Then, we can proceed as appears below

$$
\begin{aligned}
& \mathcal{L}_{I(x)}(s)=\exp \left(-\lambda \cdot p_{a} \sum_{c=-\left\lfloor d_{\max } /\left(n_{\mathrm{RB}} d_{\mathcal{A}}\right)\right\rfloor}^{\left\lceil d_{\max } /\left(n_{\mathrm{RB}} d_{\mathcal{A}}\right)\right\rceil}[\right. \\
& \mathbf{1}\left(\mu_{L}^{(1)}<\mu_{U}^{(1)}\right) \int_{t=\mu_{L}^{(1)}}^{\mu_{U}^{(1)}} \frac{s H_{\mathrm{VT}_{i}}(\tau t)^{-\alpha} \rho_{\mathrm{VT}}}{1+s H_{\mathrm{VT}_{i}}(\tau t)^{-\alpha} \rho_{\mathrm{VT}}} \mathrm{d} t \\
& \left.\left.+\mathbf{1}\left(\mu_{L}^{(2)}<\mu_{U}^{(2)}\right) \int_{t=\mu_{L}^{(2)}}^{\mu_{U}^{(2)}} \frac{s H_{\mathrm{VT}_{i}}(-\tau t)^{-\alpha} \rho_{\mathrm{VT}}}{1+s H_{\mathrm{VT}_{i}}(-\tau t)^{-\alpha} \rho_{\mathrm{VT}}} \cdot \mathrm{d} t\right]\right)
\end{aligned}
$$

where it has been applied the maximum distance to the integration limits; it has been performed the change of variables $t=y-x$; it has been expressed the absolute value function as $|t|=t \cdot \mathbf{1}(t \geq 0)-t \cdot \mathbf{1}(t<0)$ and it has been applied the indicator functions to the integration limits. Finally performing both integrals and reordering completes the proof.

Theorem 1. The ccdf of the SINR, or equivalently the capture probability, at a distance $r_{\mathrm{bc}}$ from the typical vehicle transmitter, $\mathrm{VT}_{0}$, appears below

$$
\bar{F}_{\mathrm{SINR}(x)}(\gamma)=\frac{\mathrm{e}^{-\frac{\gamma \sigma_{n}^{2}}{\rho \mathrm{VT}}\left(\tau r_{\mathrm{bc}}\right)^{\alpha}}}{d_{\mathcal{A}}} \int_{v=-\frac{d_{\mathcal{A}}}{2}}^{\frac{d_{\mathcal{A}}}{2}} \mathcal{L}_{I(x)}\left(\frac{\gamma\left(\tau r_{\mathrm{bc}}\right)^{\alpha}}{\rho_{\mathrm{VT}}}\right) \mathrm{d} v
$$

where $x=\mathrm{VT}_{0}+r_{\mathrm{bc}}$ and $\mathcal{L}_{I(x)}(s)$ is the Laplace transform of the interference, with $s=\frac{\gamma}{\rho_{\mathrm{VT}}}\left(\tau r_{\mathrm{bc}}\right)^{\alpha}$

Proof. The ccdf of the SINR at $\mathrm{x}=\mathrm{VT}_{0}+r_{\mathrm{bc}}$ can be written as

$$
\begin{aligned}
& \bar{F}_{\mathrm{SINR}(x)}(\gamma)=\operatorname{Pr}(\operatorname{SINR}(x)>\gamma) \\
& \stackrel{(\text { a) }}{=} \mathbb{E}_{\mathrm{VT}_{0}}\left[\operatorname{Pr}\left(H_{\mathrm{VT}_{0}}>\frac{\gamma}{\rho_{\mathrm{VT}}}\left(I(x)+\sigma_{n}^{2}\right)\left(\tau\left|\mathrm{VT}_{0}-x\right|\right)^{\alpha}\right)\right] \\
& \stackrel{(\mathrm{b})}{=} \frac{1}{d_{\mathcal{A}}} \int_{v=-d_{\mathcal{A}} / 2}^{d_{\mathcal{A}} / 2} \mathrm{e}^{-\frac{\gamma}{\rho_{\mathrm{VT}}} \sigma_{n}^{2}\left(\tau r_{\mathrm{bc}}\right)^{\alpha}} \mathcal{L}_{I(x)}\left(\frac{\gamma}{\rho_{\mathrm{VT}}}\left(\tau r_{\mathrm{bc}}\right)^{\alpha}\right) \cdot \mathrm{d} v
\end{aligned}
$$

where (a) comes after reordering the expression of the SINR and applying the total probability theorem over position $\mathrm{VT}_{0}$ and (b) after performing expectation over the fading and conditioning over the interference term.

Remark 1 (Exponential dependence). In view of Theorem 1, it can be observed that the capture probability only depends on $\rho_{\mathrm{VT}}$ as $c_{1} \exp \left(-c_{2}^{(1)} / \rho_{\mathrm{VT}}\right)$, which is an increasing function with respect to $\rho_{\mathrm{VT}}$, where $c_{1}$ and $c_{2}^{(1)}$ depend on other system parameters and are constants with respect to $\rho_{\mathrm{VT}}$. Analogously, the capture probability depends on the noise power, $\sigma_{n}^{2}$, as $c_{1} \exp \left(-c_{2}^{(2)} \sigma_{n}^{2}\right)$, which is a decreasing function with respect to $\sigma_{n}^{2}$.

Corollary 1. The capture probability in the limiting case where $n_{\mathrm{RB}} \rightarrow \infty$ is given as follows

$$
\begin{aligned}
& \lim _{n_{\mathrm{RB}} \rightarrow \infty} \bar{F}_{\mathrm{SINR}(x)}(\gamma)=\frac{e^{-\frac{\gamma \sigma_{n}^{2}}{\rho_{\mathrm{VT}}}\left(\tau r_{\mathrm{bc}}\right)^{\alpha}}}{d_{\mathcal{A}}} \\
& \int_{v=-\frac{d_{\mathcal{A}}}{2}}^{\frac{d_{\mathcal{A}}}{2}} \exp \left(-\lambda p_{a} \kappa\left(0, \frac{\gamma \sigma_{n}^{2}}{\rho_{\mathrm{VT}}}\left(\tau r_{\mathrm{bc}}\right)^{\alpha}, v+r_{\mathrm{bc}}\right)\right) \mathrm{d} v
\end{aligned}
$$

Proof. The proof comes after the fact when $n_{\mathrm{RB}} \rightarrow \infty$ the indicator function given in (7), $\mathbf{1}\left(y \in \mathfrak{b}_{x}\left(d_{\max }\right)\right)$, is non zero only for $c=0$.

Remark 2 (Intra-segment interference limited regime). In view of Corollary 1 it can be observed that the capture 
probability when $n_{\mathrm{RB}}$ tends to infinity is limited by the interference of the probe segment $(c=0)$, which is related to those cases where an interfering vehicle is transmitting in the same segment as the probe vehicle transmitter.

It has been necessary to obtain the Laplace transform of the interference to compute the ccdf of the SINR. However the Laplace transform of the interference is also useful to obtain the average interference, which allows us to obtain insights about the proposed method. The following Lemma gives such result.

Lemma 2. The average received interference at $x$, being the probe segment centered at the origin can be expressed as appears below

$$
\begin{aligned}
& \mathbb{E}[I(x)]=\frac{\lambda p_{a} \rho_{\mathrm{VT}}}{\alpha-1} \sum_{c=-\left\lfloor d_{\max } /\left(n_{\mathrm{RB}} d_{\mathcal{A}}\right)\right\rfloor}^{\left\lceil d_{\max } /\left(n_{\mathrm{RB}} d_{\mathcal{A}}\right)\right\rceil} \sum_{j \in\{1,2\}} \\
& \quad\left(\mu_{L}^{(j)}(c, x)\left(\mu_{U}^{(j)}(c, x)\right)^{\alpha}-\tau^{-\alpha}\left(\mu_{U}^{(j)}(c, x)\right)^{1-\alpha}\right. \\
& \quad \times \mathbf{1}\left(\mu_{L}^{(j)}(c, x)<\mu_{U}^{(j)}(c, x)\right)
\end{aligned}
$$

Proof. Using the fact that the Laplace transform can be used as a moment generating function, the average interference can be written as $\mathbb{E}[I(x)]=-\left|\frac{\mathrm{d}}{\mathrm{d} s} \mathcal{L}_{I(x)}(s)\right|_{s=0}$. Hence the proof consists on obtaining the derivative of (5) and then particularizing for $s=0$.

Remark 3 (Convergence of the interference). In view of (12) it can be stated that the average interference is only finite for $x \notin \mathfrak{b}_{c \cdot n_{\mathrm{RB}} \cdot d_{\mathcal{A}}}\left(d_{\mathcal{A}} / 2\right)$, since for $x \in \mathfrak{b}_{c \cdot n_{\mathrm{RB}} \cdot d_{\mathcal{A}}}\left(d_{\mathcal{A}} / 2\right)$ we have $\mu_{U}^{(2)}(c, x)=0$ which makes the average interference tend to infinity.

Besides the capture probability, another paramount performance metric for system design is the average BR. This result is given in the following Lemma.

Lemma 3. The average $B R$ at a distance $r_{\mathrm{bc}}$ from the typical vehicle transmitter, $\mathrm{VT}_{0}$, appears below

$$
\mathbb{E}[\operatorname{BR}(x)]=\frac{b_{w}}{n_{\mathrm{RB}}} \cdot \log _{2}(1+\gamma) \cdot \bar{F}_{\mathrm{SINR}}(\gamma)
$$

where $x=\mathrm{VT}_{0}+r_{\mathrm{bc}}$.

Proof. The proof consists on performing expectation over (3) and realizing that $\mathbb{E}[\mathbf{1}(\operatorname{SINR}(x)>\gamma)]$ is the ccdf of the SINR.

Remark 4 (Average rate when $n_{\mathrm{RB}}$ tends to infinity). In view of Lemma 3 and Corollary 1 it can be stated that for a finite SINR threshold, $\gamma$, the average BR tends to 0 as $n_{\mathrm{RB}}$ tends to infinity.

Proof. The proof consist on realizing that the ccdf of the SINR is equal or smaller than 1 , hence for a finite $\gamma$ the term $n_{\mathrm{RB}}$ in the denominator of (13) makes the average BR tend to 0.
TABLE I

Simulation PARAMETERS

\begin{tabular}{cccc}
\hline Parameter & Value & Parameter & Value \\
\hline$\lambda\left(\right.$ vehicles $\left./ \mathrm{m}^{2}\right)$ & $1 / 40$ & $p_{\mathrm{a}}$ & 0.25 \\
\hline$n_{\mathrm{RB}}$ & $\{1,3,6,100,300\}$ & $d_{\mathcal{A}}(\mathrm{m})$ & 10 \\
\hline$\rho_{\mathrm{VT}}(\mathrm{dBm} / \mathrm{Hz})$ & -30 & $b_{w}(\mathrm{MHz})$ & 9 \\
\hline$\alpha$ & 3.8 & $\tau$ & 2.6 \\
\hline$d_{\max }(\mathrm{km})$ & 6 & $\sigma_{n}^{2}(\mathrm{dBm} / \mathrm{Hz})$ & -165 \\
\hline
\end{tabular}

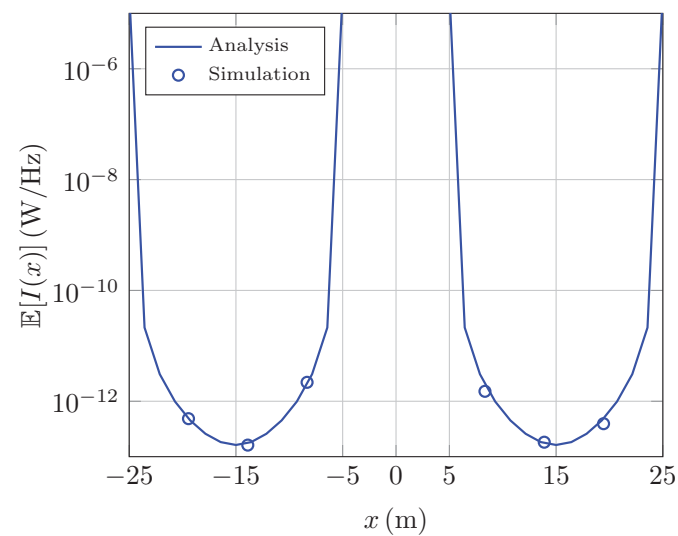

Fig. 3. Average interference received at $x$ with $n_{\mathrm{RB}}=3$. Analytical results are represented with solid lines whereas simulation results are represented with marks.

Obtained results are evaluated numerically in next section in order to illustrate the benefits of DLOC.

\section{NUMERICAL RESULTS}

In this section analytical results are compared with simulations in order to assess DLOC performance. Simulations are carried out averaging over $10^{4}$ spatial realizations. It is considered that VTs transmit with $-30 \mathrm{dBm} / \mathrm{Hz}$, a thermal noise power of $-174 \mathrm{dBm} / \mathrm{Hz}$ and a noise figure of $9 \mathrm{~dB}$, hence $\sigma_{n}^{2}=-165 \mathrm{dBm} / \mathrm{Hz}$. The parameter $d_{\max }$ is chosen as the minimum distance in which the average received power from a transmitting vehicle is a times below the noise floor, $\sigma_{n}^{2}$. Thus we have $d_{\max } \geq \frac{1}{\tau}\left(\frac{\rho_{\mathrm{VT}}}{\sigma_{n}^{2}} a\right)^{\frac{1}{\alpha}}$. With aforementioned parameters and $a=10, d_{\max }$ should be higher than $4341 \mathrm{~m}$. Simulation parameters are summarized in Table I. Through this section analytical results are drawn with solid lines whereas markers are used for simulation results.

The average interference at $x$ is illustrated in Fig. 3 for the case of $n_{\mathrm{RB}}=3$. It can be observed that average interference diverges for $x \notin \mathfrak{b}_{c \cdot n_{\mathrm{RB}} \cdot d_{\mathcal{A}}}\left(d_{\mathcal{A}} / 2\right)$ as it is stated in Remark 3. It can be noticed that the average interference has a periodic behavior along the $x$ axis, where minimum interference is achieved at the mid point between co-channel segments. To understand this fact, let us remark that, in this case, there is a segment within the interval $[-5,5]$, another within $[-35,-25]$, another within $[25,35]$, etc. Hence mid points between segments, i.e. $x=-15$ or $x=15$, are points 


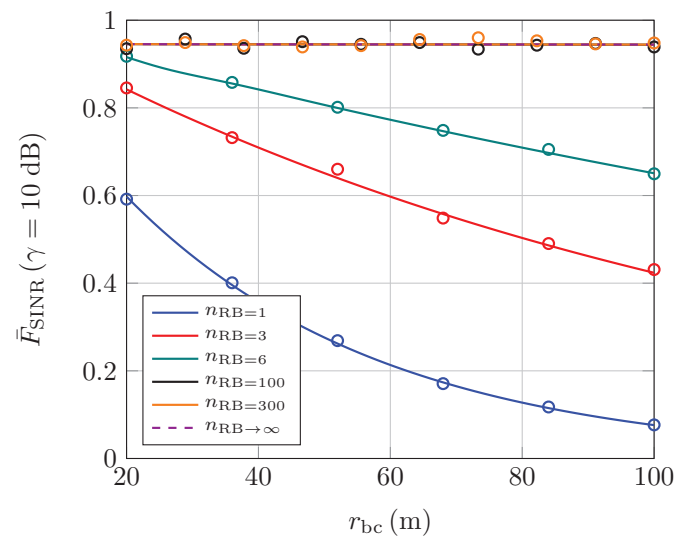

Fig. 4. ccdf of the SINR versus $r_{\mathrm{bc}}$ for $\gamma=10 \mathrm{~dB}$ with $n_{\mathrm{RB}}=$ $\{1,3,6,100,300\}$.

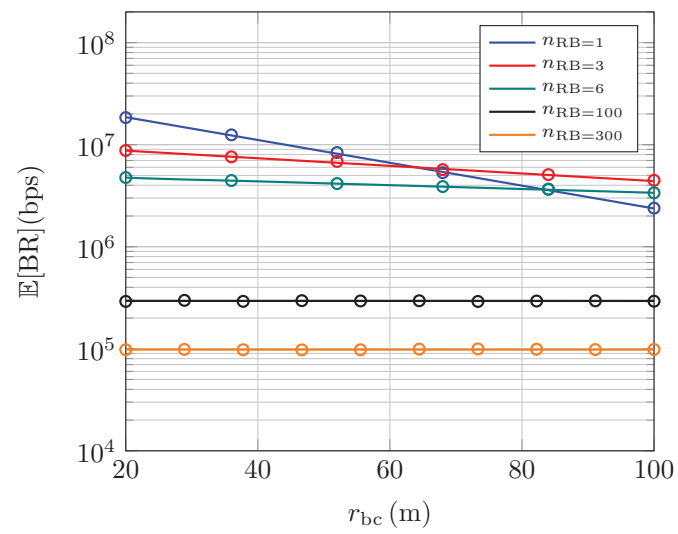

Fig. 5. Average $\mathrm{BR}$ versus $r_{\mathrm{bc}}$ for $\gamma=10 \mathrm{~dB}$ with $n_{\mathrm{RB}}=$ $\{1,3,6,100,300\}$.

that maximize the distance to interfering vehicles leading to minimal average interference.

Fig. 4 represents the ccdf of the SINR, i.e. capture probability, versus the broadcast distance $r_{\mathrm{bc}}$ for a SINR threshold of $10 \mathrm{~dB}$. The case of $n_{\mathrm{RB}}=1$ actually corresponds to the case where all VTs transmit using all the available bandwidth and interfere each other in each transmission. Interestingly, it is observed that DLOC allows to greatly increase the reliability with the design parameter $n_{\mathrm{RB}}$. This is due to the fact that the minimum co-channel distance is augmented with $n_{\mathrm{RB}}$. In particular, capture probability with $n_{\mathrm{RB}}=100$ is quite close to the maximum, which is given in Corollary 1 for $n_{\mathrm{RB}} \rightarrow \infty$. Such a maximum capture probability is around 0.95 for a broadcast distance of $100 \mathrm{~m}$ and is related to the intra-segment interference as stated in Remark 2. It can be noticed a rapid increment in the capture probability as $n_{\mathrm{RB}}$ increases for $n_{\mathrm{RB}}$ between 1 and 6 . From $n_{\mathrm{RB}}=100$ and onward, the capture probability is already very close to the upper bound.

The average BR versus the broadcast distance, $r_{\mathrm{bc}}$, is illustrated in Fig. 5 for $\gamma=10 \mathrm{~dB}$ and different number of RBs. Fig. 4 and Fig. 5 illustrate the existing trade off between reliability and $\mathrm{BR}$ : having $n_{\mathrm{RB}}=100$ achieves capture probabilities around 0.95 at a distance of $100 \mathrm{~m}$ whereas with $n_{\mathrm{RB}}=1$ capture probability is smaller than 0.1 ; however the cost to pay is that the average BR is about one order of magnitude smaller in the former case. This trade-off is also observed in the limit when $n_{\mathrm{RB}} \rightarrow \infty$ with Remarks 2 and 4 since although $n_{\mathrm{RB}} \rightarrow \infty$ leads to maximal capture probability it also leads to minimal average BR. Nevertheless, increasing $n_{\mathrm{RB}}$ does not necessarily mean that the average BR is reduced. This is because of the trade off that exists between increasing the SINR in statistical terms and increasing the bandwidth allocated to each transmission. Augmenting $n_{\mathrm{RB}}$ increases the SINR statistically; however the bandwidth used in each transmission is reduced by $n_{\mathrm{RB}}$ times. This interplay can be observed in Fig. 5 for small values of $n_{\mathrm{RB}}$, i.e. 1 to 6 . The average $\mathrm{BR}$ is higher for $n_{\mathrm{RB}}=1$ when the broadcast distance, $r_{\mathrm{bc}}$, is small, i.e. $r_{\mathrm{bc}}=20 \mathrm{~m}$; however higher distances require higher number of $\mathrm{RBs}$ to reach a better performance. In particular, the average $\mathrm{BR}$ with $n_{\mathrm{BR}}=1$ is smaller than the average $\mathrm{BR}$ with $n_{\mathrm{BR}}=3$ for $r_{\mathrm{bc}}>64$ $\mathrm{m}$, and it is smaller than the average BR with $n_{\mathrm{BR}}=6$ for $r_{\mathrm{bc}}>84 \mathrm{~m}$.

\section{Conclusions}

This paper proposes an abstraction model that allows tractable analysis for DLOC based access in vehicular networks. In such access technique vehicles access orthogonal RBs based on its geographical position in order to increase the co-channel distance. Form inspection of obtained expressions it has been identified several insights that lead to a better understanding of the studied technique. Additionally, through numerical evaluation it has been shown that: (i) the average interference depends on the receiver position, being minimal at mid points between co-channel segments; (ii) increasing the number of RBs, $n_{\mathrm{RB}}$, greatly increases the SINR in statistical terms for small values of RBs whereas the increment is minor when the number of RBs is high enough; (iii) there exist a trade off between the capture probability and BR.

\section{ACKNOWLEDGMENT}

This work has been supported by the Spanish Government (Ministerio de Economía y Competitividad) and FEDER under grants TEC2013-44442-P and TEC2016-80090-C2-1-R and by the Universidad de Málaga.

\section{REFERENCES}

[1] B. Soret, M. Gatnau Sarret, I. Z. Kovacs, F. Martin-Vega, G. Berardinelli, and N. H. Mahmood, "Radio resource management for V2V discovery," submitted to IEEE Vehicular Technology Magazine.

[2] M. Haenggi, Stochastic Geometry for Wireless Networks. Cambridge University Press, 2013

[3] B. Blaszczyszyn, P. Mhlethaler, and Y. Toor, "Maximizing throughput of linear vehicular Ad-hoc NETworks (VANETs) - a stochastic approach," in Wireless Conference, 2009. EW 2009. European, May 2009, pp. 32-36.

[4] Y. Jeong, J. W. Chong, H. Shin, and M. Z. Win, "Intervehicle Communication: Cox-Fox Modeling," IEEE Journal on Selected Areas in Communications, vol. 31, no. 9, pp. 418-433, September 2013.

[5] W. C. Cheung, T. Q. S. Quek, and M. Kountouris, "Throughput Optimization, Spectrum Allocation, and Access Control in Two-Tier Femtocell Networks," IEEE Journal on Selected Areas in Communications, vol. 30 , no. 3, pp. 561-574, April 2012. 